\title{
ELASTIC ANALYSIS OF SHEAR WALLS LOADED ANTIMETRICALLY WITH REGARD TO THEIR LONGITUDINAL CENTER LINE AND SYMMETRICALLY WITH REGARD TO THEIR TRANSVERSAL CENTER LINE PART II
}

- Equations for Conditions which are Necessary to Define the Unknown Coefficients and Unknown Factor-

\section{By Dr. MASAHIDE TOMII* and IKUO TOKUHIRO** Members of A.I.J.}

\section{NOTE}

\section{$\S 4.1$ Airy's Stress Function}

$\$ 4.2$ Unit Stresses, Unit Elongations, and Displacements in the Wall

$\S 4.3$ Stresses, Displacements, etc. in the Upper Beam and Right Column of the Frame have been reported in the previous Transactions as PART $\mathrm{I}^{4}$.

In this paper, the equations for the conditions which are necessary to define the unknown coeffcients $\left(C_{a m^{\prime}}, D_{a m^{\prime}}, A_{b n^{\prime}}, B_{b n^{\prime}}, F_{1}, F_{2}, F_{3}, F_{4}\right)$ and the unknown factor $\left(N_{b 0}\right)$, are described.

\$4.4 Equations for Conditions which are Necessary to Define the Unknown Coefficients and Unknown Factor

1. The Equations which Define the Displacements and Rotation Angle of the Shear Walls Assumed as a Rigid Body:

$$
\begin{aligned}
& \left.u\right|_{\substack{x=0 \\
y=0}}=0 \\
& \left.v\right|_{y=0}=0 .
\end{aligned}
$$

The eq. 4.56 was used to define $u_{0}$ (eq. 4.24 ) in eq. 4.17 , and the eq. 4.57 was used to define the integration constant in eq. 4.18 is equal to zero, therefore eqs. 4.56 and 4.57 are unnecessary.

2. The Equation for the Right Angle Condition of Each Corner of the Wall:

By assuming the corner joints of the frame as the rigid zone, the right angle condition is defined by the following equation.

$$
\left.\tau\right|_{\substack{x=a \\ y=b}}=0 \text {. }
$$

where, $\tau$ is given by eq. 4.7 in PART $\mathrm{I}^{4}$.

3. The Equations for the Continuity Conditions on the Boundaries between the Wall and Frame:

3-A By expanding $\left.t \sigma_{y}\right|_{y=b}$ and $w_{a 1}$ in the Fourier series with regard to $\sin \xi_{m}$ :

$$
\left.\left.t \sigma_{y}\right|_{y=b} \doteqdot t \bar{\sigma}_{y}\right|_{y=b}=t \sum_{m} \sigma_{y m} \sin \xi_{m} \cdots \cdots \cdots(4.59) \quad w_{a_{1}} \doteqdot \bar{w}_{a_{1}}=\sum_{m} w_{a_{1} m} \sin \xi_{m}
$$

and making $\left.t \bar{\sigma}_{y}\right|_{y=b}$ equal to $\bar{w}_{a_{1}}, r_{m}$ (number of $m=1,3,5, \cdots \cdots$ ) equations for the continuity of the normal stresses (per unit length) on the boundary between the wall and beam are obtained as follows (see Fig. 4.6).

$$
t \sigma_{y m}=w_{a 1 m}(\text { for each } m=1,3,5, \cdots \cdots)
$$

where,

$$
\begin{aligned}
\sigma_{y m}= & -\left(\frac{m \pi}{2 a}\right)^{2}\left(C^{\prime}{ }_{a m} \cosh \alpha_{a m}+D^{\prime}{ }_{a m} \alpha_{a m} \sinh \alpha_{a m}\right) \\
& +\sum_{n}(-1)^{\frac{m-1}{2}}(-1)^{-\frac{n}{2}}\left(\frac{n \pi}{2 b}\right)^{2}\left[A^{\prime}{ }_{b n} \frac{4 \lambda_{a} n}{\pi\left(n^{2}+\lambda_{a}{ }^{2} m^{2}\right)} \cosh \alpha_{b n}+B^{\prime}{ }_{b n}\left\{\frac{2 n^{2}}{n^{2}+\lambda_{a}{ }^{2} m^{2}} \sinh \alpha_{b n}\right.\right.
\end{aligned}
$$

* Prof. of Kyushu Univ., Dr. Eng. ** Assistant of Kyushu Univ. 


$$
\begin{aligned}
& \left.\left.+\frac{4 \lambda_{a} n\left(n^{2}+3 \lambda_{a}{ }^{2} m^{2}\right)}{\pi\left(n^{2}+\lambda_{a}^{2} m^{2}\right)^{2}} \cosh \alpha_{b n}\right\}\right] \\
& +(-1)^{\frac{m-1}{2}} \frac{12}{a^{2}}\left(\frac{2}{m \pi}\right)^{2}\left[\left(F_{1}+F_{3}\right)-2 \lambda_{b}^{2}\left(F_{1}+\lambda_{b}^{2} F_{2}\right)\left\{1-2\left(\frac{2}{m \pi}\right)^{2}\right\}\right] \\
& w_{a 1 m}=I_{a}\left(\frac{m \pi}{2 a}\right)^{5}\left[C_{a m}^{\prime}(1+\nu) \sinh \alpha_{a m}+D_{a m}^{\prime}\left\{-(1-\nu) \sinh \alpha_{a m}+(1+\nu) \alpha_{a m} \cosh \alpha_{a m}\right\}\right] \\
& +\frac{t D_{a}}{2}\left(\frac{m \pi}{2 a}\right)^{3}\left\{C_{a m}^{\prime} \sinh \alpha_{a m}+D_{a m}^{\prime}\left(\sinh \alpha_{a m}+\alpha_{a m} \cosh \alpha_{a m}\right)\right\} \\
& -\left(\frac{m \pi}{2 a}\right)\left(\frac{D_{a}}{2} q_{a 2 m}+e_{a} q_{a \epsilon m}\right)+w_{a 2 m}-(-1)^{\frac{m-1}{2}} \frac{12 t D_{a}}{a^{2} b}\left(\frac{2}{m \pi}\right)^{2} F_{1}
\end{aligned}
$$

3-B By expanding $\left.t \sigma_{x}\right|_{x=a}$ and $w_{b_{1}}$ in the Fourier series with regard to $\cos \eta_{n}$ :

$$
\left.\left.t \sigma_{x}\right|_{x=a} \doteqdot t \bar{\sigma}_{x}\right|_{x=a}=t \sum_{n} \sigma_{x n} \cos \eta_{n}+t \sigma_{x_{0}} \cdots \cdots(4.64) \quad w_{b_{1}} \doteqdot \bar{w}_{b_{1}}=\sum_{n} w_{b_{1 n}} \cos \eta_{n}+w_{b_{10}}
$$

and making $\left.t \bar{\sigma}_{x}\right|_{x=a}$ equal to $\bar{w}_{b_{1}}, r_{n}$ (number of $\left.n=2,4,6, \cdots \cdots\right)+1$ equations for the continuity of the normal stresses (per unit length) on the boundary between the wall and column are obtained as follows (see Fig. 4.6).

$t \sigma_{x n}=w_{b_{1 n}}($ for each $n=2,4,6, \cdots \cdots)$ and $t \sigma_{x 0}=w_{b_{10}}$

where,

$$
\begin{aligned}
& \sigma_{x n}=-\left(\frac{n \pi}{2 b}\right)^{2}\left(A_{b n}^{\prime} \sinh \alpha_{b n}+B_{b n}^{\prime} \alpha_{b n} \cosh \alpha_{b n}\right) \\
& +\sum_{m}(-1)^{\frac{m-1}{2}}(-1)^{-\frac{n}{2}}\left(\frac{m \pi}{2 a}\right)^{2}\left[C^{\prime} a_{m}^{\frac{4 \lambda_{b} m}{\pi\left(m^{2}+\lambda_{b}{ }^{2} n^{2}\right)}} \sinh \alpha_{a m}+D_{a m}^{\prime}\left\{\frac{4 m^{2}}{m^{2}+\grave{i}_{b}^{2} n^{2}} \cosh \alpha_{a m}\right.\right. \\
& \left.\left.+\frac{4 \lambda_{b} m\left(m^{2}+3 \lambda_{b}{ }^{2} n^{2}\right)}{\pi\left(m^{2}+\lambda_{b}^{2} n^{2}\right)} \sinh \alpha_{a m}\right\}\right]+(-1)^{-\frac{n}{2}} \frac{48}{b^{2}}\left(\frac{2}{n \pi}\right)^{2} F_{2} \\
& \sigma_{x_{0}}=(-1)^{\frac{m-1}{2}}\left(\frac{m \pi}{2 a}\right)^{2}\left[C_{a m}^{\prime}\left(\frac{2}{m \pi}\right) \lambda_{b} \sinh \alpha_{a m}+D_{a m}^{\prime}\left\{\cosh \alpha_{a m}+\left(\frac{2}{m \pi}\right) \lambda_{b} \sinh \alpha_{a m}\right\}\right] \\
& +\frac{2}{b^{2}}\left(F_{1}+2 F_{2}+F_{4}\right) \\
& w_{b i n}=I_{b}\left(\frac{n \pi}{2 b}\right)^{5}\left[A_{b n}^{\prime}(1+\nu) \cosh \alpha_{b n}+B_{b n}^{\prime}\left\{-(1-\nu) \cosh \alpha_{b n}+(1+\nu) \alpha_{b n} \sinh \alpha_{b n}\right\}\right] \\
& +\frac{t D_{b}}{2}\left(\frac{n \pi}{2 b}\right)^{3}\left\{A_{b n}^{\prime} \cosh \alpha_{b n}+B^{\prime}{ }_{b n}\left(\cosh \alpha_{b n}+\alpha_{b n} \sinh \alpha_{b n}\right)\right\}+\left(\frac{D_{b}}{2} q_{b 2 n}+e_{b} q_{b e n}\right)\left(\frac{n \pi}{2 b}\right) \\
& +w_{b_{2 n}}-(-1)^{\frac{n}{2}} \frac{24 t D_{b}}{a b^{2}}\left(\frac{2}{n \pi}\right)^{2} F_{2} \\
& w_{b_{10}}=\frac{12 I_{b}}{a b^{4}}\left\{\lambda_{a}^{2} F_{1}+2(2+\nu) F_{2}\right\}-\frac{t D_{b}}{a b^{2}}\left(3 F_{1}+2 F_{2}+F_{4}\right)+w_{b 20}
\end{aligned}
$$

3-C By expanding $\left.\varepsilon_{x}\right|_{y=b}$ and $\varepsilon_{a 1}$ in the Fourier series with regard to $\sin \xi_{m}$ :

$$
\left.\left.\varepsilon_{x}\right|_{y=b} \doteqdot \bar{\varepsilon}_{x}\right|_{y=b}=\sum_{m} \varepsilon_{x m} \sin \xi_{m} \ldots \ldots \ldots \ldots \ldots \ldots(4.71) \quad \varepsilon_{a 1}=\bar{\varepsilon}_{a 1}=\sum_{m} \varepsilon_{a 1 m} \sin \xi_{m} \cdots
$$

and making $\left.\bar{\varepsilon}_{x}\right|_{y=b}$ equal to $\bar{\varepsilon}_{a 1}, r_{m}$ (number of $m=1,3,5, \cdots \cdots$ ) equations for the continuity of the unit elongations parallel to and on the boundary between the wall and beam are obtained as follows (see Fig. 4.7).

$\varepsilon_{x m}=\varepsilon_{a 1 m}($ for each $m=1,3,5, \cdots \cdots)$

where,

$$
\begin{aligned}
& \varepsilon_{x m}=\frac{1}{E}\left(\frac{m \pi}{2 a}\right)^{2}\left[C_{a m}^{\prime}(1+\nu) \cosh \alpha_{a m}+D_{a m}^{\prime}\left\{2 \cosh \alpha_{a m}+(1+\nu) \alpha_{a m} \sinh \alpha_{a m}\right\}\right] \\
& -\sum_{n}(-1)^{\frac{m-1}{2}}(-1)^{\frac{n}{2}}\left(\frac{n \pi}{2 b}\right)^{2}\left[A^{\prime}{ }_{b n} \frac{4 \lambda_{a} n(1+\nu)}{\pi\left(n^{2}+\lambda_{a}{ }^{2} m^{2}\right)} \cosh \alpha_{b n}+B^{\prime}{ }_{b n}\left\{\frac{2 n^{2}(1+\nu)}{n^{2}+\lambda_{a}{ }^{2} m^{2}} \sinh \alpha_{b n}\right.\right. \\
& \left.\left.+\frac{4 \lambda_{a} n\left\{(1+3 \nu) \lambda_{a}{ }^{2} m^{2}-(1-\nu) n^{2}\right\}}{\pi\left(n^{2}+\lambda_{a}^{2} m^{2}\right)^{2}} \cosh \alpha_{b n}\right\}\right]+(-1)^{\frac{m-1}{2}} \frac{4}{a^{2}}\left(\frac{2}{m \pi}\right)^{2}\left[\lambda_{b}^{2}\left(6 F_{2}+F_{4}\right)\right. \\
& \left.-3 \nu\left(F_{1}+F_{3}\right)+3 \lambda_{b}{ }^{2}\left\{F_{1}+2 \nu\left(F_{1}+\lambda_{b}{ }^{2} F_{2}\right)\right\}\left\{1-2\left(\frac{2}{m \pi}\right)^{2}\right\}\right]
\end{aligned}
$$




$$
\varepsilon_{a 1 m}=\frac{1}{E}\left(\begin{array}{rl}
\frac{l_{a}}{Z_{a}}\left(\frac{m}{2} \frac{\pi}{a}\right)^{3}\left[C_{a m}^{\prime}(1+\nu) \sinh \alpha_{a m}+D_{a m}^{\prime}\left\{-(1-\nu) \sinh \alpha_{a m}+(1+\nu) \alpha_{a m} \cosh \alpha_{a m}\right\}\right] \\
\quad-\frac{t}{A_{a}}\left(\frac{m \pi}{2 a}\right)\left\{C_{a m}^{\prime} \sinh \alpha_{a m}+D_{a m}^{\prime}\left(\sinh \alpha_{a m}+\alpha_{a m} \cosh \alpha_{a m}\right)\right\}-\frac{1}{A_{a}}\left(\frac{2 a}{m \pi}\right)\left(q_{a 2 m}+q_{a e m}\right) \\
& -(-1)^{\frac{m-1}{2}} 2\left(\frac{2}{m \pi}\right)^{2}\left[\frac{12 I_{a}}{Z_{a} a^{2} b}\left\{\nu F_{1}+2\left(F_{1}+\lambda_{b}{ }^{2} F_{2}\right)\right\}+\frac{2}{A_{a}} \frac{t}{b}\left(2 F_{2}+F_{4}\right)\right. \\
& \left.+\frac{a}{A_{a}}\left(q_{a 20}+q_{a e n}\right)+\frac{6}{A_{a}} \frac{t}{b} F_{1}\left\{1-2\left(\frac{2}{m \pi}\right)^{2}\right\}\right]
\end{array}\right.
$$

3-D By expanding $\left.\varepsilon_{y}\right|_{x=a}$ and $\varepsilon_{b_{1}}$ in the Fouries series with regard to $\cos \xi_{n}$ :

$$
\left.\left.\varepsilon_{y}\right|_{x=a} \doteqdot \bar{\varepsilon}_{y}\right|_{x=a}=\sum_{n} \varepsilon_{y n} \cos \eta_{i n}+\varepsilon_{y_{0}} \ldots \ldots \ldots \ldots \ldots \text { (4.76) } \quad \varepsilon_{b_{1}}=\bar{\varepsilon}_{b_{1}}=\sum_{n} \varepsilon_{b_{1 n}} \cos \eta_{n}+\varepsilon_{b_{10}} \text {. }
$$

and making $\left.\bar{\varepsilon}_{y}\right|_{x=a}$ equal to $\bar{\varepsilon}_{b_{1}}, r_{n}$ (number of $\left.n=2,4,6, \cdots \cdots\right)+1$ equations for the continuity of the unit elongations parallel to and on the boundary between the wall and column are obtained as follows (see Fig. 4.7).

$\varepsilon_{y n}=\varepsilon_{b_{1 n} n}($ for each $n=2,4,6, \cdots)$ and $\varepsilon_{y_{0}}=\varepsilon_{b_{10}}$

where,

$$
\begin{aligned}
& \varepsilon_{y n}=\frac{1}{E}\left(\left(\frac{n \pi}{2 b}\right)^{2}\left[A_{b n}^{\prime}(1+\nu) \sinh \alpha_{b n}+B_{b n}^{\prime}\left\{2 \sinh \alpha_{b n}+(1+\nu) \alpha_{b n} \cosh \alpha_{b n}\right\}\right]\right. \\
& -\sum_{m}(-1)^{\frac{m-1}{2}}(-1)^{\frac{n}{2}}\left(\frac{m \pi}{2 a}\right)^{2}\left[C_{a m}^{\prime} \frac{4 \lambda_{b} m(1+\nu)}{\pi\left(m^{2}+\lambda_{b}{ }^{2} n^{2}\right)} \sinh \alpha_{a m}\right. \\
& \left.D^{\prime} a m\left\{\frac{2 m^{2}(1+\nu)}{m^{2}+\lambda_{b}{ }^{2} n^{2}} \cosh \alpha_{a m}+\frac{4 \lambda_{b} m\left\{(1+3 \nu) \lambda_{b}{ }^{2} n^{2}-(1-\nu) m^{2}\right\}}{\pi\left(m^{2}+\lambda_{b}{ }^{2} n^{2}\right)^{2}} \sinh \alpha_{a m}\right\}\right] \\
& \because(-1)^{\frac{n}{2}} \frac{24}{b^{2}}\left(\lambda a^{2} F_{1}-2 \nu F_{2}\right)\left(\frac{2}{n \pi}\right)^{2} \\
& \varepsilon_{y_{0}}=\frac{1}{E}\left(-\sum_{m}(-1)^{\frac{m-1}{2}}\left(\frac{m \pi}{2 a}\right)^{2}\left[C_{a m}^{\prime}(1+\nu)\left(\frac{2}{m \pi}\right) \lambda_{b} \sinh \alpha_{a m}+D_{a m}^{\prime}\left\{(1+\nu) \cosh \alpha_{a m}\right\}\right.\right. \\
& \left.\left.-(1-\nu)\left(\frac{2}{m \pi}\right) \lambda_{b} \sinh \alpha_{a m}\right\}\right]+\frac{2}{b^{2}}\left[\lambda_{a}^{2}\left\{3 F_{3}-2 \lambda_{b}^{2}\left(F_{1}+\lambda_{b}^{2} F_{2}\right)\right\}\right. \\
& \left.-\nu\left(F_{1}+F_{4}\right)+\lambda_{a}^{2} F_{1}-2 \nu F_{2}\right] \\
& \varepsilon_{b 1 n}=\frac{1}{E}\left(\frac{I_{b}}{Z_{b}}\left(\frac{n \pi}{2 b}\right)^{3}\left[A_{b n}^{\prime}(1+\nu) \cosh \alpha_{b n}+B_{b n}^{\prime}\left\{-(1-\nu) \cosh \alpha_{b n}+(1+\nu) \alpha_{b n} \sinh \alpha_{b n}\right\}\right]\right. \\
& -\frac{t}{A_{b}}\left(\frac{n \pi}{2 b}\right)\left\{A_{b n}^{\prime} \cosh \alpha_{b n}+B_{b n}^{\prime}\left(\cosh \alpha_{b n}+\alpha_{b n} \sinh \alpha_{b n}\right)\right\} \\
& +\frac{1}{A_{b}}\left(\frac{2 b}{n \pi}\right)\left(q_{b 2 n}+q_{b e n}\right)+(-1)^{\frac{n}{2}} 4\left(\frac{2}{n \pi}\right)^{2}\left[-\frac{6 I_{b}}{Z_{b} a b^{2}}\left\{\lambda_{a}{ }^{2} F_{1}+2(2+\nu) F_{2}\right\}\right. \\
& \left.-\frac{1}{A_{b}} \frac{t}{a}\left(3 F_{1}+F_{4}\right)+\frac{2}{A_{b}} \frac{t}{a} F_{2}\left\{1-6\left(\frac{2}{m \pi}\right)^{2}\right\}\right] \\
& \varepsilon_{b_{10}}=\frac{1}{E}\left(\frac{-2 I_{b}}{Z_{b} a b^{2}}\left\{\left(\lambda_{a}^{2}+3 \nu\right) F_{1}-2\left(3 \lambda_{b}^{2}-2-\nu\right) F_{2}+3 \lambda_{a}^{2} F_{3}+(2+\nu) F_{4}\right\}\right. \\
& -\frac{1}{A_{b}} \frac{t}{a}\left(F_{1}+\frac{1}{5} F_{2}+\frac{1}{3} F_{4}\right)+\frac{N_{b_{0}}}{A_{b}}
\end{aligned}
$$

4. The equations for the Equilibrium for the Forces in the $y$-direction and Moments on the joint of the Beam and Column:

$\Sigma Y=0$ on the joint :

$$
-\left.N_{b}\right|_{y=b}+\left.Q_{a}\right|_{x=a}+V_{0}=0
$$

$\Sigma M=0$ on the joint :

$$
\left.M_{a}\right|_{x=a}-\left.M_{b}\right|_{y=b}+\left.\frac{D_{b}}{2} Q_{a}\right|_{x=a}
$$

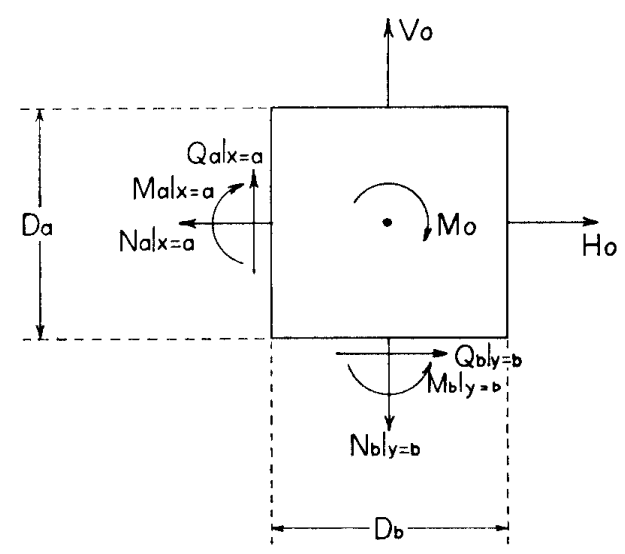

Fig. 4.4 The forces and moments on the joint of the beam and column 


$$
-\left.\frac{D_{a}}{2} Q_{b}\right|_{y=b}+M_{0}=0
$$

On the quarter shear wall,

i. the total of the external forces in the $x$-direction (see eq. 4.1)

ii. the normal stress $t \sigma_{x}$ per unit length on the section $x=0$ of the wall and the axial force $N_{a}$ on the cross section $x=0$ of the beam

iii. the shearing stress $t \tau$ per unit length in the $x$ direction on the section $y=0$ of the wall and the shearing force $Q_{b}$ on the cross section $y=0$ of the column

are all zero, therefore $\Sigma X=0$ is satisfied identically as eq. 4.1. The equations $\Sigma X=0, \Sigma Y=0$ and $\Sigma M=0$ on any part of the wall, beam, and column are satisfied in this analysis. Therefore, $\Sigma X=0$ on the joint which is the remaining part of the quarter shear wall is satisfied identically and so unnecessary.

\section{Equations for Conditions which are Necessary to Define} the Unknown Coefficients and Unknown Factor

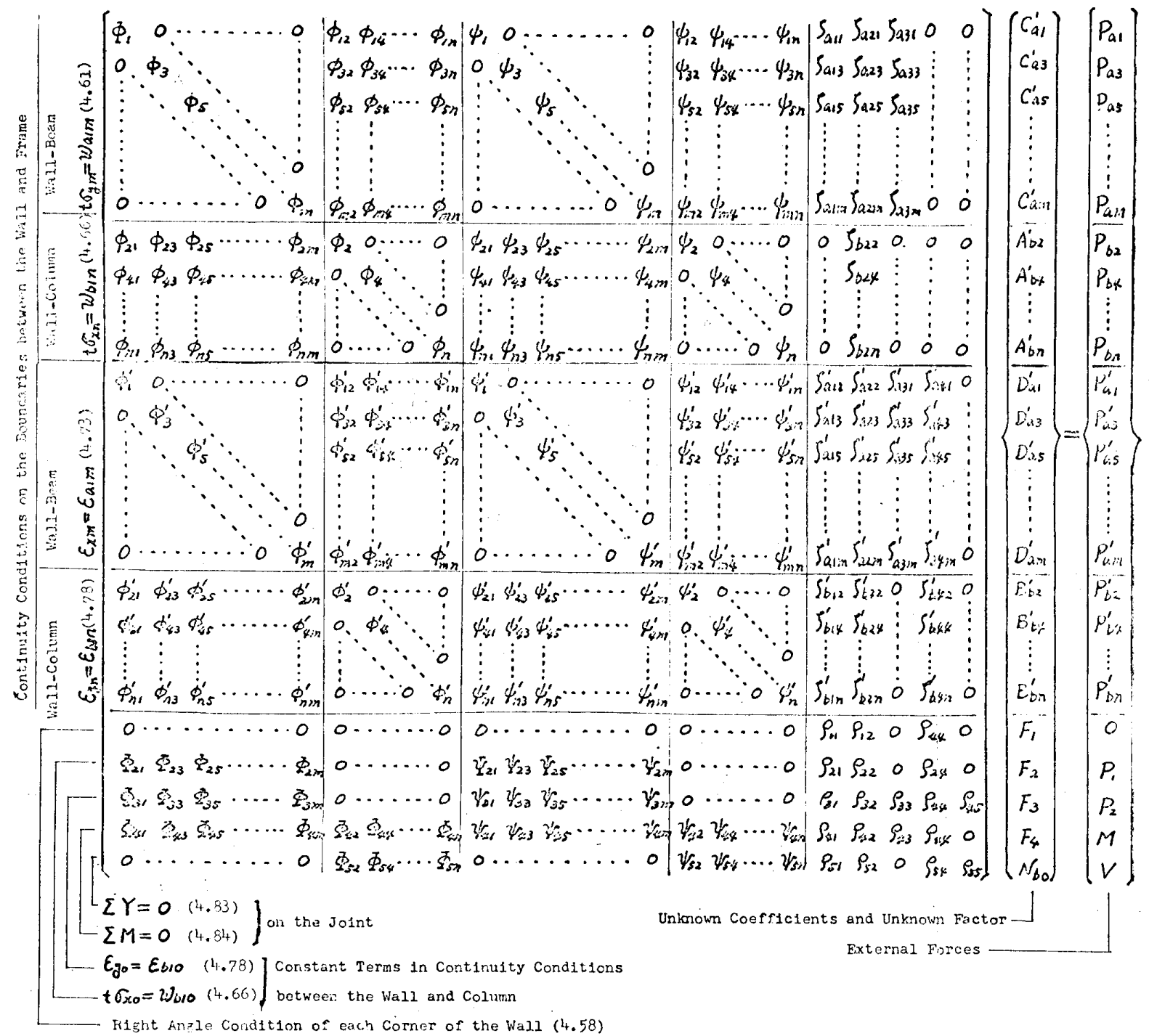

where,

$$
\begin{aligned}
\phi_{m}= & -\left(\frac{m \pi}{2}\right)^{2} \cosh \alpha_{a m}-\frac{I_{a}}{a^{3} t}\left(\frac{m \pi}{2}\right)^{5}(1+\nu) \sinh \alpha_{a m}-\frac{D_{a}}{2 a}\left(\frac{m \pi}{2}\right)^{3} \sinh \alpha_{a m} \\
\phi_{m}= & -\left(\frac{m \pi}{2}\right)^{2} \alpha_{a m} \sinh \alpha_{a m}-\frac{I_{a}}{a^{3} t}\left(\frac{m \pi}{2}\right)^{5}\left\{-(1-\nu) \sinh \alpha_{a m}+(1+\nu) \alpha_{a m} \cosh \alpha_{a m}\right\} \\
& -\frac{D_{a}}{2 a}\left(\frac{m \pi}{2}\right)^{3}\left(\sinh \alpha_{a m}+\alpha_{a m} \cosh \alpha_{a m}\right) \\
\phi_{m n}= & (-1)^{\frac{m-1}{2}}(-1)^{\frac{n}{2}} \lambda_{b}{ }^{2}\left(\frac{n \pi}{2}\right)^{2} \frac{4 \lambda_{a} n}{\pi\left(n^{2}+\lambda_{a}^{2} m^{2}\right)} \cosh \alpha_{b n}
\end{aligned}
$$


$\psi_{m n}=(-1)^{\frac{m-1}{2}}(-1)^{\frac{n}{2}} \lambda_{b}^{2}\left(\frac{n \pi}{2}\right)^{2}\left[\frac{2 n^{2}}{n^{2}+\lambda_{a}^{2} m^{2}} \sinh \alpha_{b n}+\frac{4 \lambda_{a} n\left(n^{2}+3 \lambda_{a}^{2} m^{2}\right)}{\pi\left(n^{2}+\lambda_{a}^{2} m^{2}\right)^{2}} \cosh \alpha_{b n}\right]$

$\phi_{n}=-\left(\frac{n \pi}{2}\right)^{2} \sinh \alpha_{b n}-\frac{I_{b}}{b^{3} t}\left(\frac{n \pi}{2}\right)^{5}(1+\nu) \cosh \alpha_{b n}-\frac{D_{b}}{2 b}\left(\frac{n \pi}{2}\right)^{3} \cosh \alpha_{b n}$

$\psi_{n}=-\left(\frac{n \pi}{2}\right)^{2} \alpha_{b n} \cosh \alpha_{b n}-\frac{I_{b}}{b^{3} t}\left(\frac{n \pi}{2}\right)^{5}\left\{-(1-\nu) \cosh \alpha_{b n}+(1+\nu) \alpha_{b n} \sinh \alpha_{b n}\right\}$

$-\frac{D_{b}}{2 b}\left(\frac{n \pi}{2}\right)^{3}\left(\cosh \alpha_{b n}+\alpha_{b n} \sinh \alpha_{b n}\right)$

$\phi_{n m}=(-1)^{\frac{m-1}{2}}(-1)^{\frac{n}{2}} \lambda_{a}^{2}\left(\frac{m \pi}{2}\right)^{2} \frac{4 \lambda_{b} m}{\pi\left(m^{2}+\lambda_{b}^{2} n^{2}\right)} \sinh \alpha_{a m}$

$\phi_{n m}=(-1)^{\frac{m-1}{2}}(-1)^{\frac{n}{2}} \lambda_{a}^{2}\left(\frac{m \pi}{2}\right)^{2}\left[\frac{4 m^{2}}{m^{2}+\lambda_{b}^{2} n^{2}} \cosh \alpha_{a m}+\frac{4 \lambda_{b} m\left(m^{2}+3 \lambda_{b}^{2} n^{2}\right)}{\pi\left(m^{2}+\lambda_{b}^{2} n^{2}\right)^{2}} \sinh \alpha_{a m}\right]$

$\phi^{\prime}{ }_{m}=\left(\frac{m \pi}{2}\right)^{2}(1+\nu) \cosh \alpha_{a m}-\frac{I_{a}}{Z_{a} a}\left(\frac{m \pi}{2}\right)^{3}(1+\nu) \sinh \alpha_{a m}+\frac{a t}{A_{a}}\left(\frac{m \pi}{2}\right) \sinh \alpha_{a m}$

$\phi_{m}^{\prime}=\left(\frac{m \pi}{2}\right)^{2}\left\{2 \cosh \alpha_{a m}+(1+\nu) \alpha_{a m} \sinh \alpha_{a m}\right\}-\frac{I_{a}}{Z_{a} a}\left(\frac{m \pi}{2}\right)^{3}\left\{-(1-\nu) \sinh \alpha_{a m}\right.$

$\left.+(1+\nu) \alpha_{a m} \cosh \alpha_{a m}\right\}+\frac{a t}{A_{a}}\left(\frac{m \pi}{2}\right)\left(\sinh \alpha_{a m}+\alpha_{a m} \cosh \alpha_{a m}\right)$

$\phi^{\prime}{ }_{m n}=-(-1)^{\frac{m-1}{2}}(-1)^{\frac{n}{2}} \lambda_{b}^{2}\left(\frac{n \pi}{2}\right)^{2}(1+\nu) \frac{4 \lambda_{a} n}{\pi\left(n^{2}+\lambda_{a}^{2} m^{2}\right)} \cosh \alpha_{b n}$

$\phi_{m n}^{\prime}=-(-1)^{\frac{m-1}{2}}(-1)^{\frac{n}{2}} \lambda_{b}^{2}\left(\frac{n \pi}{2}\right)^{2}\left[\frac{2 n^{2}(1+\nu)}{n^{2}+\lambda_{a}^{2} m^{2}} \sinh \alpha_{b n}+\frac{4 \lambda_{a} n\left\{(1+3 \nu) \lambda_{a}^{2} m^{2}-(1-\nu) n^{2}\right\}}{\pi\left(n^{2}+\lambda_{a}^{2} m^{2}\right)^{2}} \cosh \alpha_{b n}\right]$

$\phi_{n}^{\prime}=\left(\frac{n \pi}{2}\right)^{2}(1+\nu) \sinh \alpha_{b n}-\frac{I_{b}}{Z_{b} b}\left(\frac{n \pi}{2}\right)^{3}(1+\nu) \cosh \alpha_{b n}+\frac{b t}{A_{b}}\left(\frac{n \pi}{2}\right) \cosh \alpha_{b n}$

$\psi_{n}^{\prime}=\left(\frac{n \pi}{2}\right)^{2}\left\{2 \sinh \alpha_{b n}+(1+\nu) \alpha_{b n} \cosh \alpha_{b n}\right\}-\frac{I_{b}}{Z_{b} b}\left(\frac{n \pi}{2}\right)^{3}\left\{-(1-\nu) \cosh \alpha_{b n}+(1+\nu) \alpha_{b n} \sinh \alpha_{b n}\right\}$ $+\frac{b t}{A_{b}}\left(\frac{n \pi}{2}\right)\left(\cosh \alpha_{b n}+\alpha_{b n} \sinh \alpha_{b n}\right)$

$\phi_{n m}^{\prime}=-(-1)^{\frac{m-1}{2}}(-1)^{\frac{n}{2}} \lambda_{a}{ }^{2}\left(\frac{m \pi}{2}\right)^{2} \frac{4 \lambda_{b} m(1+\nu)}{\pi\left(m^{2}+\lambda_{b}{ }^{2} n^{2}\right)} \sinh \alpha_{a m}$

$\phi_{n m}^{\prime}=-(-1)^{\frac{m-1}{2}}(-1)^{\frac{n}{2}} \lambda_{a}^{2}\left(\frac{m \pi}{2}\right)^{2}\left[\frac{2 m^{2}(1+\nu)}{m^{2}+\lambda_{b}^{2} n^{2}} \cosh \alpha_{a m}+\frac{4 \lambda_{b} m\left\{(1+3 \nu) \lambda_{b}^{2} n^{2}-(1-\nu) m^{2}\right\}}{\pi\left(m^{2}+\lambda_{b}^{2} n^{2}\right)^{2}} \sinh \alpha_{a m}\right]$

$\zeta_{a 1 m}=12(-1)^{\frac{m-1}{2}}\left(\frac{2}{m \pi}\right)^{2}\left[1+\frac{D_{a}}{b}-2 \lambda_{b}^{2}\left\{1-2\left(\frac{2}{m \pi}\right)^{2}\right\}\right]$

$\zeta_{a 2 m}=-24(-1)^{\frac{m-1}{2}}\left(\frac{2}{m \pi}\right)^{2} \lambda_{b}{ }^{4}\left\{1-2\left(\frac{2}{m \pi}\right)^{2}\right\}, \quad \zeta_{a 3 m}=12(-1)^{\frac{m-1}{2}}\left(\frac{2}{m \pi}\right)^{2}$

$P_{a m}=-\left(\frac{m \pi}{2}\right) \frac{a}{t}\left(\frac{D_{a}}{2} q_{a 2 m}+e_{a} q_{a e m}\right)+\frac{a^{2}}{t} w_{a 2 m}, \quad \zeta_{b 2 n}=48(-1)^{\frac{n}{2}}\left(\frac{2}{n \pi}\right)^{2}\left(1+\frac{D_{b}}{2 a}\right)$

$P_{b n}=\left(\frac{n \pi}{2}\right) \frac{b}{t}\left(\frac{D_{b}}{2} q_{b 2 n}+e_{b} q_{b e n}\right)+\frac{b^{2}}{t} w_{b_{2 n}}$

$\zeta_{a 1 m}^{\prime}=12(-1)^{\frac{m-1}{2}}\left(\frac{2}{m \pi}\right)^{2}\left[\lambda_{b}\left(\lambda_{b}+2 \nu \lambda_{b}+\frac{a t}{A_{a}}\right)\left\{1-2\left(\frac{2}{m \pi}\right)^{2}\right\}-\nu+2 \frac{I_{a}}{Z_{a} b}(2+\nu)\right]$

$\zeta_{a 2 m}^{\prime}=8(-1)^{\frac{m-1}{2}}\left(\frac{2}{m \pi}\right)^{2} \lambda_{b}\left[3 \nu \lambda_{b}^{3}\left\{1-2\left(\frac{2}{m \pi}\right)^{2}\right\}+3 \lambda_{b}+6 \lambda_{b} \frac{I_{a}}{Z_{a} b}+\frac{a t}{A_{a}}\right]$

$\zeta_{a 3 m}^{\prime}=-12(-1)^{\frac{m-1}{2}} \nu\left(\frac{2}{m \pi}\right)^{2}, \quad \zeta_{a 4 m}^{\prime}=4(-1)^{\frac{m-1}{2}} \lambda_{b}\left(\frac{2}{m \pi}\right)^{2}\left(\lambda_{b}+\frac{a t}{A_{a}}\right)$

$P_{a m}^{\prime}=-\frac{a^{3}}{A_{a}}\left(\frac{2}{m \pi}\right)\left\{\left(q_{a 2 m}+q_{a e m}\right)+2(-1)^{\frac{m-1}{2}}\left(q_{a 20}+q_{a e_{0}}\right)\right\}$

$\zeta_{b 1 n}^{\prime}=12(-1)^{\frac{n}{2}}\left(\frac{2}{n \pi}\right)^{2} \lambda_{a}\left(2 \lambda_{a}+\frac{2 I_{b}}{Z_{b} a} \lambda_{a}+\frac{b t}{A_{b}}\right)$

$\zeta_{b 2 n}^{\prime}=-8(-1)^{\frac{n}{2}}\left(\frac{2}{n \pi}\right)^{2}\left[\lambda_{a} \frac{b t}{A_{b}}\left\{1-6\left(\frac{2}{m \pi}\right)^{2}\right\}+6\left\{\nu-\frac{I_{b}}{Z_{b} a}(2+\nu)\right\}\right]$

$\zeta_{b 4 n}^{\prime}=4(-1)^{\frac{n}{2}}\left(\frac{2}{n \pi}\right)^{2} \lambda_{a} \frac{b t}{A_{a}}, \quad P_{b n}^{\prime}=\frac{b^{3}}{A_{b}}\left(\frac{2}{n \pi}\right)\left(q_{b 2 n}+q_{b e n}\right)$ 


$$
\begin{aligned}
& \rho_{11}=3.0, \quad \rho_{12}=2.0, \quad \rho_{14}=1.0, \quad \Phi_{2 m}=(-1)^{\frac{m-1}{2}} \lambda_{a}\left(\frac{m \pi}{2}\right) \sinh \alpha_{a m} \\
& \psi_{2 m}=(-1)^{\frac{m-1}{2}} \lambda_{a}{ }^{2}\left(\frac{m \pi}{2}\right)^{2}\left[\cosh \alpha_{a m}+\left(\frac{2}{m \pi}\right) \lambda_{b} \sinh \alpha_{a m}\right] \\
& \rho_{21}=2-12 \lambda_{a}{ }^{2} \frac{I_{b}}{a b^{2} t}+\frac{3 D_{b}}{a}, \quad \rho_{22}=2\left\{2+\frac{D_{b}}{a}-12(2+\nu) \frac{I_{b}}{a b^{2} t}\right\}, \quad \rho_{24}=2+\frac{D_{b}}{a}, \quad P_{1}=\frac{b^{2}}{t} w_{b 20} \\
& \Phi_{3 m}=(-1)^{\frac{m-1}{2}} \lambda_{a}(1+\nu)\left(\frac{m \pi}{2}\right) \sinh \alpha_{a m} \\
& \psi_{3 m}=(-1)^{\frac{m-1}{2}} \lambda_{a}{ }^{2}\left(\frac{m \pi}{2}\right)^{2}\left[(1+\nu) \cosh \alpha_{a m}-(1-\nu)\left(\frac{2}{m \pi}\right) \lambda_{a} \sinh \alpha_{a m}\right] \\
& \rho_{31}=2(2+\nu)-2 \lambda_{a}{ }^{2}-\lambda_{a} \frac{b t}{A_{b}}-\frac{2 I_{b}}{Z_{b} a}\left(\lambda_{a}^{2}+3 \nu\right) \\
& \rho_{32}=4\left(\lambda_{b}{ }^{2}+\nu\right)-\frac{4 I_{b}}{Z_{b} a}\left(2+\nu-3 \lambda_{b}{ }^{2}\right)-\frac{\lambda_{a} b t}{5 A_{b}}, \quad \rho_{33}=-6 \lambda_{a}{ }^{2}\left(1+\frac{I_{a}}{Z_{b} a}\right), \quad \rho_{34}=2 \nu-2(2+\nu) \frac{I_{b}}{Z_{b} a}-\frac{\lambda_{a} b t}{3 A_{b}} \\
& \rho_{35}=\frac{b^{2}}{A_{b}}, \quad \Phi_{4 m}=(-1)^{\frac{m-1}{2}}\left(\frac{m \pi}{2}\right)^{3} \frac{I_{a}}{a^{3} t}(1+\nu) \sinh \alpha_{a m}, \quad \Phi_{4 n}=(-1)^{\frac{n}{2}}\left(\frac{n \pi}{2}\right)^{3} \frac{I_{b}}{b^{3} t}(1+\nu) \cosh \alpha_{b n} \\
& \psi_{4 m}=(-1)^{\frac{m-1}{2}}\left(\frac{m \pi}{2}\right)^{3} \frac{I_{a}}{a^{3} t}\left\{-(1-\nu) \sinh \alpha_{a m}+(1+\nu) \alpha_{a m} \cosh \alpha_{a m}\right\} \\
& \psi_{4 n}=(-1)^{\frac{n}{2}}\left(\frac{n \pi}{2}\right)^{3} \frac{I_{b}}{b^{3} t}\left\{-(1-\nu) \cosh \alpha_{b n}+(1+\nu) \alpha_{b n} \sinh \alpha_{b n}\right\} \\
& \rho_{41}=\frac{12 I_{a}}{a^{2} b t}(2+\nu)\left(1+\frac{D_{b}}{2 a}\right)+\frac{6 I_{b}}{a b^{2} t}\left\{\nu+\lambda_{a}^{2}\left(1+\frac{D_{a}}{2 b}\right)\right\} \\
& \rho_{42}=-\frac{12 \lambda_{b}{ }^{2} I_{a}}{a^{2} b t}\left(2+\frac{D_{b}}{2 a}\right)-\frac{12 I_{b}}{a b^{2} t}\left\{\lambda_{b}^{2}-(2+\nu)\left(1+\frac{D_{a}}{b}\right)\right\}, \quad \rho_{43}=\frac{6 \lambda_{a}{ }^{2} I_{b}}{a b^{2} t}, \quad \rho_{44}=2(2+\nu) \frac{I_{b}}{a b^{2} t} \\
& M=-\frac{M_{0}}{t}+\frac{D_{b}}{2 t}\left(\frac{D_{a}}{2} q_{a 20}+e_{a} q_{a e 0}\right), \quad \Phi_{5 n}=-(-1)^{-\frac{n}{2}}\left(\frac{n \pi}{2}\right) \frac{t}{b} \cosh \alpha_{b n} \\
& \psi_{5 n}=-(-1)^{\frac{n}{2}}\left(\frac{n \pi}{2}\right) \frac{t}{b}\left(\cosh \alpha_{b n}+\alpha_{b n} \sinh \alpha_{b n}\right), \quad \rho_{51}=-3 \frac{t}{a}+\frac{12 I_{a}}{a^{3} b}(2+\nu) \\
& \rho_{52}=-\frac{t}{a}+\frac{24 I_{a}}{a^{3} b} \lambda_{b}^{2}, \quad \rho_{54}=-\frac{t}{a}, \quad \rho_{55}=1.0 \\
& V=V_{0}-\sum_{n}(-1)^{\frac{n}{2}}\left(\frac{2 b}{n \pi}\right)\left(q_{b_{2 n}}+q_{b e n}\right)-\left(\frac{D_{a}}{2} q_{a 20}+e_{a} q_{a e 0}\right)
\end{aligned}
$$

\section{NOTE}

As the pages are limited,

$\$ 4.5$ Numerical Results of an Example

\section{ACKNOWLEDGEMENT \\ REFERENCES}

will be reported in the following Transactions as PART III. 


\title{
縦軸に関して逆対称，横軸に関して対称な外力を 受ける耐震壁の弾性解析 第 2 報
}

\author{
（未知係数および末知積分定数を求めるための条件式）
}

$\begin{array}{lllll}\text { 正会員 } & \text { 富 } & \text { 井 } & \text { 政 } & \text { 英* } \\ \text { 正会貝 } & \text { 德 } & \text { 広 } & \text { 育 } & \text { 夫** }\end{array}$

\section{序}

次の項目は「緃軸に関して逆対称，横軸に関して対称 な外力を受ける耐震壁の弾性解析 第 1 報」としてすで に発表した4。

$\$ 4.1$ エアリーの応力関数

$\S 4.2$ 壁板の応力度, ひずみ度, 変位

$\$ 4.3$ 上ばり, 右柱の灾力, 変位, その他

本論では $\$ 4.1, \S 4.2, \S 4.3$ に示した応力度，ひず み度, 応力, 変位などの各式に含まれているフーリエ級 数および代数関数の未知保数揖よび未知積分定数を求め るための条件式を示す。

§4.4 未知係数および末知積分定数を求めるための 条件式 (連立方程式)

1. 耐震壁の剛体としての変位および回転を規定する 条件式

耐震壁の中心（原点 $x=0, y=0$ ) の水平変位を零と規 定することを 4.56 式で示し， $y=0$ の線上で垂直変位 を零と規定することを 4.57 式で示した。 4.56 式は壁 板の水平変位（既報 4.17 式）の積分定数 $u_{0}$ を求める ためにすでに使用し(4.24 式)，4.57 式は壁板の垂直変 位 (既報) 4.18 式) の積分定数を零と置くときの条件 としてすでに使用したので, 本項の条件式としては不要 である。

2. 壁板四隅の直角条件式

付帯ラーメンの節点を剛域と仮定することにより，壁 板四隅は常に直角を保っことになり，その直角条件式を 4.58 式で示した。ての一般式は既報4) 4.7 式に示した。

3. 壁板と付帯ラーメンの連続条件式

3-A) 壁板とはりの境界上の垂直応力 $\left.t \sigma_{y}\right|_{y=b}$ (既報 ${ }^{4}$ 4.4 式）および $w_{a_{1}}$ (既報) 4.36 式）をそれぞれ $\sin$ $\xi_{m}$ に関するフーリエ級数に展開し（4.59 式および 4.60 式参照)，両者を等值すると，各 $m=1,3,5 \cdots$ の $\sin \xi_{m}$ に関し，その倸数 $t \sigma_{y m}$ 拉よび $w_{a 1 m}$ を等值する条件 式 (4.61 式) が $m=1,3,5 \cdots$ の数 $r_{m}$ 個だけ作られる。 $\sigma_{y m}$ および $w_{a 1 m}$ はそれぞれ 4.62 式および 4.63 式で

$*$ 九州大学教授・工㥀 $* *$ 同 助手

(昭利 44 年 6 月 2 日本㯖受理・討綸期限昭和 45 年 3 月末日)
与えられる。

3-B） 壁板とはりの境界上の垂直応力 $\left.t \sigma_{x}\right|_{x=a}$ (既報) 4.6 式）および $w_{b_{1}}$ (既報) 4.51 式）をそれぞれ $\cos$ $\eta_{n}$ に関するフーリエ級数に展開し（4.64 式および 4.65 式参照)，両者を等值すると，各 $n=2,4,6, \cdots$ の $\cos \eta_{n}$ に関し，その係数 $t \sigma_{x n}$ および $w_{b_{1 n}}$ を等值する条件式 $r_{n}$ 個, $t \sigma_{x_{0}}$ と $w_{b_{10}}$ を等值する条件式 1 個, 合計 $r_{n}+$ 1 個の条件式 (4.66 式) が作られる。 $\sigma_{x n}, \sigma_{x 0}, w_{b_{1 n}}$, $w_{b_{10}}$ はそれぞれ 4.67 式，4.68 式，4.69 式，4.70 式 で与えられる。

3-C）壁板とはりの境界上の境界線方向伸縮ひずみ 度 $\left.\varepsilon_{x}\right|_{y=b}$ (既報 ${ }^{4)} 4.13$ 式) および $\varepsilon_{a 1}$ (既報 ${ }^{4} 4.38$ 式) をそれぞれ $\sin \xi_{m}$ に関するフーリエ級数に展開し（4.71 式および 4.72 式参照)，両者を等值すると，各 $m=1$, $3,5, \cdots の \sin \xi_{m}$ に関しその係数 $\varepsilon_{x m}$ および $\varepsilon_{a 1 m}$ を 等値する条件式が $m=1,3,5, \cdots$ の数 $r_{m}$ 個だけ作られ る。 $\varepsilon_{x m}$ および $\varepsilon_{a 1 m}$ はそれぞれ 4.74 式および式 4.75 で与えられる。

3-D）壁板と柱の境界上の境界線方向ひずみ度 $\left.\varepsilon_{y}\right|_{x=a}$ (既報) 4.15 式）および $\varepsilon_{b_{1}}$ (既報) 4.53 式）をそれ ぞれ $\cos \eta_{n}$ に関するフーリ工級数に展開し（4.76 式お よび 4.77 式参照), 両者を等值すると, 各 $n=2,4,6$, $\cdots の \cos \eta_{n}$ に関し，その係数 $\varepsilon_{y n}$ および $\varepsilon_{b_{1 n}}$ を等值 する条件式が $n=2,4,6, \cdots$ の数 $r_{n}$ 個と，定数項 $\varepsilon_{y_{0}}$ と $\varepsilon_{b_{10}}$ を等值する条件式 1 個, 合計 $r_{n}+1$ 個の条件式 (4.78 式) が作られる。 $\varepsilon_{y n}, \varepsilon_{y 0}, \varepsilon_{b_{11}}, \varepsilon_{b_{10}}$ はそれぞれ 4.79 式，4.80 式， 4.81 式， 4.82 式で与えられる。

4. 付帯ラーメンの節点 (剛域) における軸方向力およ びモーメントの验合条件式

節点 (剛域)に扝ける $y$ 方向の釣合条件式 $\Sigma Y=0$ は 4.83 式で, モーメントの釣合式は 4.84 式で与えられ る。

耐震壁の既知面内外力は $x$ 方向の釣合条件を満たすよ らに与えられており，任意の值をとることが許されない (既報 ${ }^{4}$ の序文参照)。耐震壁の 4 分の $1(x, y$ が正の範 囲)の部分について,

i. $x$ 方向の外力の合計 (4.1 式参照) 
ii. $x=0$ の断面上の壁板の 垂直応力 $\left.t \sigma_{x}\right|_{x=0}$ および はりの軸方向力 $\left.N_{a}\right|_{x=0}$

iii. $y=0$ の断面上の壁板のせん断応力 $\left.t \tau\right|_{y=0}$ および 柱のせん断力 $\left.\boldsymbol{Q}_{b}\right|_{y=0}$

がいずれも0であることから， $x$ 方向に関する力の釣合 式は 4.1 式と一致し, 恒等的に $x$ 方向の力の釣合が成 立する。壁板，はり，柱のいかなる部分も $\Sigma X=0, \Sigma Y$ $=0, \Sigma M=0$ が成立するように解析をすすめている。 ゆえに耐震壁の 4 分の 1 の残りの部分である付帯ラーメ ンの節点 (剛域) も $\Sigma X=0$ が成立することになり，
$\Sigma X=0$ の条件式を必要としない。

本項で述べた必要な条件式をまとめ，フーリエ級数お よび代数関数の未知係数の各項, 外力の各項をそれぞれ マトリックスで一括表示した (本文参照)。 おことわり 紙面の都合で次の項目は第 3 報として続報する。 $\S 4.5$ 数值計算例 謝辞 参考文献

注 : 文中の式および図は英論文を参照されたい。 
U.D.C. $69.022: 699.84: 624.043$

\section{ELASTIC ANALYSIS OF SHEAR WALLS LOADED ANTIMETRICALLY WITH REGARD TO THEIR LONGITUDINAL CENTER LINE AND SYMMETRICALLY WITH REGARD TO THEIR TRANSVERSAL} CENTER LINE - Part II - Equations for Conditions which are Necssary to Define the Unknown Coefficeients and Unknown Factor (See Page 43)

by Dr. MASAHIDE TOOMII Prof. of Kyushu Univ., and IKUO TOKUHIRO, Assistant of Kyushu Univ, Members of A.I,J.

U.D.C. $624.073 .1: 539.385$

\section{ELASTO-PLASTIC TORSION OF THIN-WALLED OPEN SECTIONS}

by MAMORU KIMURA, Research Officer, Takenaka Technical Research Laboratory and Dr. TOSHIRO SUZUKI, Associate Proffesor, Nagoya Institute of Technology.

This paper describes the torsional behaviour of thin-walled open sections, and deals with pure torsion and combined torsion with initial axial stress of bendins moment. The narrow rectangular plate is chosen for this analysis and following assumptions are intraduced.

(1) The field of small deflection is considered.

(2) The cross section is underformed and remains plane during torsion.

(3) Statical behaviour of open section is equivalent to that of the gatharing of very thin-walled closed sections.

(4) The yield criferion of the material is based on Von. Mises' law, and work-hardening is not taken into account.

The approximate solution is obtained by nomographs for the combined torsion. The computed results are compared with the experimental results and they show good agreement under pure torsion. In case of torsion combined bending, the experimental result is investigated in early stage of twisting angle, because, when the initial bending about major axis is great, the specimen produces lateral deformation about minor axis with increasing angle of twist.

U.D.C. $624.078: 624.071 .3 / 072.2$

\section{LIMIT ANALYSIS OF BEAM-COLUMN CONNECTIONS (VII-2)}

-Connections of Wide-Flange Beam to Box-Column subjected to Bending and Thrust-

(See Page 57)

by Dr. HISASHI TANAKA, Prof., Inst. of Indu. Science Univ. of Tokyo, TSUNEO SHIGENOBU, Assist. Inst. of Indu. Sci. Univ. of Tokyo. Members of A.I.J. 